\title{
The use of eclipses in the evaluation of absolute stellar information
}

\author{
Edwin Budding ${ }^{1} \dagger$, Denis Sullivan ${ }^{2}$ \\ and Michael Rhodes ${ }^{3}$ \\ ${ }^{1}$ Carter National Observatory of New Zealand, PO Box 2909, Wellington, New Zealand \\ email: ebudding@comu.edu.tr \\ ${ }^{2}$ Victoria University of Wellington, Wellington, New Zealand \\ ${ }^{3}$ Brigham Young University, Provo, Utah, USA.
}

\begin{abstract}
Eclipsing binary light curves have provided the 'royal road' (Russell 1948) to fundamental astrophysical information on stars. If radial velocities of the components and a reliable colour/temperature/flux relation are available, parallaxes may be determined and compared with direct measures, e.g. by Hipparcos. Accuracies of existing measures are considered and aspects of the development of this and other methods of distance determination reviewed. Roles for multiwavelength techniques (e.g. VLBA, broad-spectrum photometry) are noted. The recovery of information on new planets orbiting remote stars by transit phenomena will be looked into within this context.
\end{abstract}

\section{Introduction}

The fitting of physical models to photometric data - particularly, in the stellar context, sets of relative flux measures at discrete phases (light curves) - concerns how information in the data maps into the parameters: sizes, masses, luminosities, and notably, for this meeting, distances, that scientists are seeking. Henry Norris Russell (1948) said that eclipsing binary stars offer a 'royal road' for such purposes, and although this route to parallax determinations has been increasingly recognized, particularly since the work of Lacy (1978), it may not be as direct to this particular destination as to some others.

\section{Photometric calibration}

The 31.572126 magnitudes by which the Sun's apparent magnitude would increase if moved to a distance of $10 \mathrm{pc}$ carries the same accuracy as that of the A.U. However, the actual value of $m_{v}(\odot)=-26.75$ is still hardly known to better than four decimal figures for various technical reasons. These include bringing a body as unique as the Sun, from the terrestrial point of view, on to the same scale of measurement as applies to other stars, and the lengthy, interdependent framework of comparison measurements needed to build up a particular magnitude scale or system. Still, four decimal digits is a relatively high accuracy in the context of stellar parallaxes.

At $10 \mathrm{pc}$, the Sun's apparent magnitude would also be its absolute magnitude (4.82), which, folded with a standard photopic transmission curve, comes to $2.94 \times 10^{-8}$ lux or $4.32 \times 10^{-11} \mathrm{~W} \mathrm{~m}^{-2}$. Comparison of normalized transmission curves for standard optical filters then leads to the zero point of, say, Johnson's $V$ scale as $3.08 \times 10^{-9} \mathrm{~W} \mathrm{~m}^{-2}$.

$\dagger$ Present address: Physics Dept., 18 March University of Canakkale, TR 17020, Turkey. 
Received fluxes $f$ can be directly measured by a normal astronomical photometer, generally as a number of photon-events per second, and, especially if the photometer behaves linearly and the zero point of the relevant scale is known, such counts can be directly transferred to watts per square meter. Typically, after taking account of various absorption effects and response efficiencies, when using a regular telescope set up for photometry, measured optical fluxes are on the order of $\sim 10^{-10}$ to $10^{-12} \mathrm{~W} \mathrm{~m}^{-2}$ for primary standard stars (although not normally given in such units). But notice that these fluxes, if transmitted across empty space, are simply related to surface fluxes $F$ at the photospheres of the measured stars by

$$
f=F R^{2} / r^{2},
$$

where $R$ is the radius of the measured star and $r$ is its distance. Hence, the parallax, $1 / r$ $(\mathrm{pc})=4.433 \times 10^{7} \sqrt{f / F} / R_{\odot}$, is determined if we can obtain the ratio of measured to surface flux and the radius of the star, here expressed in solar units.

The most direct way to obtain surface fluxes is to use Eq. (2.1) again, but with the ratio $R / r$ as half the angular diameter, combining a measurement of that with $f$. Angular diameters may be found by interferometry, or sometimes, at least for some zodaical stars, by a rather special kind of eclipse, namely occultations by the hard edge of the Moon, particularly in IR light. The derivation of surface fluxes, or corresponding temperatures, has become a focus for such angular diameter and apparent magnitude measurement. If stellar parallaxes become known, as they notably have been in the wake of the Hipparcos programme (ESA 1997), corresponding radii are determinable.

The known behaviour of continuum radiation from models of normal photospheres allows us to expect a fairly simple connection between surface flux and colour. Empirical forms have received attention, particularly since the work of Barnes \& Evans (1976), and if such a form can be supported, for example, through angular diameter and apparent magnitude checks, it can be applied to parallax determinations when the radii of the stars are independently known. This is the case for eclipsing binary stars that have good quality light and radial velocity curves and it forms the basis of the 'eclipse method'.

Let us first rewrite (2.1) for the parallax $\Pi$, following usual terminology (e.g. Popper 1998):

$$
\log \Pi=7.450-\log R-0.2 m_{V}-2 F_{V}^{\prime}
$$

where $F_{V}^{\prime}=0.25 \log F_{V}, F_{V}$ being the surface flux as in (2.1) in the Johnson $V$ system. This expression is related to the effective surface temperature $T_{e}$ by

$$
F_{V}^{\prime}=\log T_{e}+0.1 \mathrm{BC}
$$

where $\mathrm{BC}$ is the bolometric correction $m_{b o l}-m_{V}$, connecting visual and total-radiation magnitude scales.

The dependence of flux on colour, or form of $F_{V}^{\prime}=F(B-V)$, found by Popper (1998) is well represented by the following:

$$
\begin{aligned}
& \quad F_{V}^{\prime}(\text { early })=3.957-0.311(B-V)+0.586(B-V)^{2}-5.713(B-V)^{3}, \\
& (-0.2<B-V \leqslant 0.1) ; \text { and } \\
& \quad F_{V}^{\prime}(\text { late })=3.958-0.361(B-V)+0.103(B-V)^{2}-0.076(B-V)^{3} \\
& (0.1<B-V \leqslant 1.2) .
\end{aligned}
$$




\section{Eclipsing binary stars}

Russell's original light curve 'solution' procedure lacked the sophistication of presentday modelling, although a couple of points may be worth noting. Thus, (i) the introduction of more elaborate theoretical models, by itself, does not deal with the underlying issues of curve-fitting adequacy, uniqueness and determinacy and (ii) Russell and his students were concerned with the application of the results of light curve analysis to stars in general. Emphasis in more recent work often concerns the special peculiarities of close binaries themselves. Interactive evolution, for example, has engendered its own detailed research and the development of relevant physics. But stellar models suitable for general parameter evaluation (including parallaxes), to data-limited accuracies, of detached pairs were available in the wake of Kopal's (1959) 'classical' monograph.

Kopal's approach utilized well-known spherical harmonic expansions for the component interactions. These are formally integrable and thus able to characterize light variations to the same order of approximation as the distortion of figure and in a comparable number of manageable terms. Kopal (1959) also dealt with surfaces of zero velocity and limiting dynamic stability within close binaries, pointing out the relatively tractable form of the 'Roche' approximation, when internal density distributions are neglected. This has proved useful in photometric studies of close binaries. Depending on quadrature accuracy, when components become relatively very close, with radii expressed as a fraction of the orbital separation $R / A>\sim 0.35$, representations of surfaces based on this approximation may become closer to actual physical distortions than those using series approximations with finite density distribution that stop after terms in $(R / A)^{5}$ (as in Kopal's original presentation). While numerical integration approaches are much more demanding in computer time, that is becoming increasingly less of an issue. User-friendly software packages, such as Bradstreet's Binary Maker (based on the approach of Wilson \& Devinney (W-D) 1971), allow such models to be easily compared with data or those of other approaches.

Close binary system models for the light variations require the separate specification of around 16 independent physical quantities (cf. Budding 1993). The effects these parameters have on the light curve shape are, however, sometimes quite similar to each other, so that, in a deterministic sense, they become interdependent. A typical close system light curve would be insufficient, by itself, to furnish all physical parameters, although one could normally expect to make fair estimates for most, if not all, of the more basic geometric ones, using reasonable assumptions about the others.

It is important to remember this interdependence of parameters in light curve analysis. The relative radius of the primary component $r_{1}$, for example, i.e. the mean spherical radius divided by the mean orbital separation, is derived from eclipse photometry. While published findings often quote values to three or more significant decimal digits, a twodigit value is already rather optimistic for the likely information content of many light curves, if we admit to the correlated effects of uncertainty in all the other parameters. The extent of disagreement between different sources for even the more well-known examples suggests two significant figures is, conservatively, realistic, though a third digit is usually included.

\section{Practical tests}

The application of eclipsing binary data to the direct checking of basic stellar parameters was the subject of a number of seminal publications of D. M. Popper. The review already cited (Popper 1998) examined the properties of 14 well-studied systems within 
$125 \mathrm{pc}$ with low scatter Hipparcos parallaxes. The thrust of that paper was towards establishing the flux-colour relation for Main Sequence (MS)-like stars and the most commonly used filters $(V$ and $B)$, for which purpose the choice of relatively near stars rendered insignificant the potential complication of interstellar absorption. The procedure can then be inverted by using (2.2) to test the agreement of individual parallaxes. We show the results in Fig. 1.

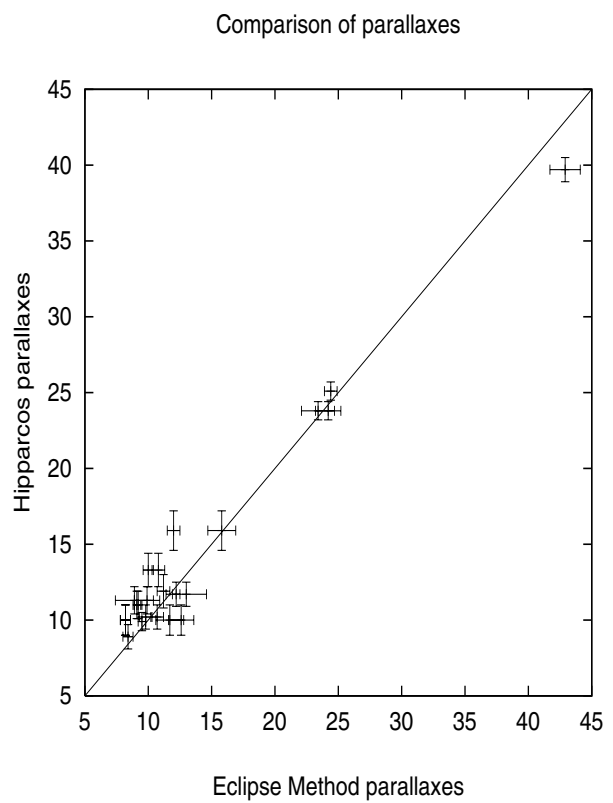

Figure 1. Comparison of Hipparcos parallaxes (in mas) with those from eclipsing binaries containing stars of known magnitudes, sizes and surface fluxes derived from their $B-V$ colours using Popper's (1998) data.

The high-parallax star in the upper right of Fig. 1, playing an important role in establishing the correlation across the range of values, though with some apparent departure from prediction, is $\beta$ Aur. This is one of the closest of a small number of eclipsing binaries with measured angular diameters, light and radial velocity curve solutions, and direct measurements of parallax. Nordström \& Johansen (1994) provided a detailed review of this system, in which they gave a parallax of $40.3 \pm 0.4$ mas, confirmed by interferometry and cross-checked by several other methods. This is within its own error limit of the Hipparcos parallax $(39.7 \pm 0.8$ mas $)$. Nordström \& Johansen give radii of primary and secondary components as 2.77 and $2.63 \mathrm{R}_{\odot}$, to a claimed $1 \%$ accuracy. The angular diameters of the two stars, from these values, are 1.04 and 0.99 mas, i.e. somewhat less than the cited average measurement of 1.05 mas, which yields an average temperature of $9120 \mathrm{~K}$. The decreased average radius implies a slightly hotter average, in essential agreement with the $9280 \mathrm{~K}$ average from Nordström \& Johansen.

The $V$ magnitudes of the two components, using Nordström \& Johansen's adopted luminosity ratio and the SIMBAD listed combined value, are 2.587 and 2.713. Corresponding $B-V$ values are 0.061 and 0.092 . Substitution in the above equations then yields 40.9 and 42.8 mas for the parallaxes. These may be compared with a similar treatment by Semeniuk (2000), who used Lacy's (1979) adopted form of $F_{V}^{\prime}=F(V-R)$, and derived a mean parallax of 46.6 mas. With the Popper flux-colour relation, the primary appears almost within the error limit of the Hipparcos parallax, though the secondary is 
distinctly less in agreement. But it is worth noting, as did Semeniuk (2000), that $\beta$ Aur has eclipses that are only $0.1 \mathrm{mag}$ deep and for which, therefore, we should not expect parameters to have the same determinacy as more typical examples. We note that slightly larger radii, particularly for the secondary, would reduce the disparities, both for the angular diameter measurement and the flux-dependent parallax.

A number of points lower down the correlation appear rather discordant in the sense of the photometric parallaxes being larger than Hipparcos values. These turn out to be the 'RS CVn' binaries SZ Psc, LX Per, RT And and UV Psc. Light curves of the latter two were intensively studied by a group at the University of New Mexico at Albuquerque, using the 'spot-cleaning' technique of Budding \& Zeilik (1987). These systems contain stars that are quite similar to the Sun, except that they are rotating $\sim 30-40$ times faster. Since, from (2.2), parallaxes can be increased by decreasing radii, it is tempting to think that these may have been overestimated in the analyses. Even if we push the radii down to their reasonably lowest limits, however, the parallax disparity remains, and we should look towards the more influential flux term to seek an explanation. In fact, as Popper (1998) noticed, the existence of dark photospheric spots would reduce the mean surface flux, while not changing the apparent colour. This can be easily checked from the apparent temperatures that go with the angular diameters: only $5100 \mathrm{~K}$ for the G5 primary of UV Psc, for example. This is an interesting point in relation to the spot-cleaning. That technique dealt only with the differential, cyclically varying component of maculation and not directly with its steady, phase-independent component. That component would reduce mean flux estimates of stars like RT And and UV Psc, even with cleaned light curves, below what could be estimated from the flux-colour relation of a spot-free photosphere. The extent of the parallax disparity suggests the steady component of the darkening is quite appreciable: up to $30 \%$ of the total luminosity would be affected, or $\sim 3$ times greater than typical 'spot-wave' components.

Semeniuk (2000) considered 19 stars from a homogeneous sample of 47 eclipsing binaries studied by Lacy (1979) that includes 8 of the binaries in Popper's (1998) review. These stars all had relatively low Hipparcos errors, usually of around $10 \%$ or less. We show a similar sample in Fig. 2, that includes 7 of the Semeniuk stars not in Popper's list, a further 2 stars (YZ Cas and KW Hya) for which very good eclipsing binary data exist (cf. Malkov 1993; http://simbad.u-strasbg.fr) and also the binary HD 209458 (see Section 6, below). We have also placed the Sun, as if seen at the distance of $25 \mathrm{pc}$, in the same figure. The uncertainty of the photometric parallax is here reflecting uncertainties of the solar magnitudes in the $B V$ system and the derived stellar flux calibration.

Semeniuk (2000) concluded that a higher accuracy of distance modulus was possible, using such well-known binaries, than would follow from the Hipparcos parallaxes, provided certain stars were excluded. As with Popper, she found the RS CVn stars systematically low in mean surface flux values. She also proposed rejecting the Algol binaries $\beta$ Per and $\delta$ Lib from her high-accuracy list. Although these stars have experienced interactive evolution histories, their primaries, at least, show photospheric conditions resembling those of normal MS stars. The results of applying new solution data indicate a satisfactory accord with Hipparcos parallaxes and this is potentially advantageous for reasons considered below. Details on all 24 reference binaries considered in this paper are presented in Table 1.

\section{Algols and checks on distances}

Algols are the most commonly observed type of eclipsing binary. The reason is the selection effect of a relatively deep primary minimum, caused by the primary's occultation 


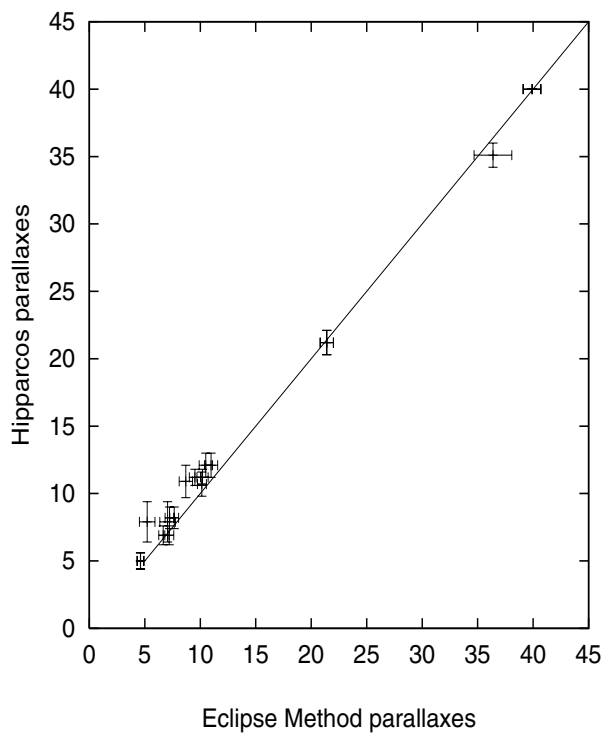

Figure 2. Comparison of Hipparcos parallaxes with 10 eclipsing binaries, mainly from Semeniuk's (2000) list.

by a cool, subgiant secondary. As well as favouring their easy recognition, this circumstance facilitates their discovery in surveys of remote systems, such as the neighbouring galaxies. Another interesting consequence of the constitution of many of these binaries is that the rapidly rotating secondaries show 'activity' characteristics in their convective envelopes. This means that, among other things, we can expect Algols to be microwave sources, and existing data indeed show radio characteristics similar to those of RS CVn stars (Slee et al. 1987). Hence, this opens up Algols to the positional finesse of modern high-precision radio-interferometric techniques. Mutel et al.'s (1998) VLBI map showing coronal features around the secondary of $\beta$ Per was also giving support to less-direct positional information from the optical domain on the system's geometry and parallax (cf. also Lestrade et al. 1999). It would be very interesting to check if such features, resolved for $\beta$ Per, could be made out for some other nearby Algols.

Another, still relatively near, Algol is $\delta$ Lib: a close binary made up of A0V + K0IV stars that has been the subject of a recent intensive study (Budding et al. 2004a) combining photometric, spectroscopic and radio information. Like $\beta$ Per, $\delta$ Lib seems to be in a gravitationally bound system with 3, or more stars. Suspicions of such arrangements often come from data on the times of minimum light, which are again facilitated by deep eclipses. For $\delta$ Lib, more precise times of minima could check on a tentative 13 min amplitude oscillation indicated in recent photoelectric eclipse times. This would allow estimates of the semi-major axis, period and eccentricity parameters of a third-body orbit. Detailed light curve analysis, with relatively high determinacy, of wide-spectrum photometry including the infra-red light curves of Lazaro et al. (2002), gave persuasive evidence of a late A or early $\mathrm{F}$ type companion having a feasible (MS) mass of $\sim 1.4 \mathrm{M}_{\odot}$.

Extra stars in a light source produce complications for the Hipparcos reduction procedures, so various issues are interrelated for binaries with wider orbit companions and it is worthwhile to recheck the astrometry. Worek (2001) already postulated a low mass companion for $\delta$ Lib to account for irregularities in the primary's radial velocity 
Table 1. Parameters of 24 binaries (and Sun) used to compare Eclipse Method and Hipparcos parallaxes.

\begin{tabular}{|c|c|c|c|c|c|c|c|}
\hline Star & $\mathrm{R}$ & V & $F_{V}^{\prime}$ & $\Pi_{\mathrm{EM}}$ & err. & $\Pi_{\text {Hip }}$ & err. \\
\hline$\zeta$ Phe A & 2.85 & 4.221 & 4.032 & 12.2 & 0.3 & 11.7 & 0.8 \\
\hline$\zeta$ Phe B & 1.85 & 5.591 & 3.975 & 13.0 & 1.6 & 11.7 & 0.8 \\
\hline$\beta$ Aur & 2.49 & 2.643 & 3.947 & 42.9 & 1.2 & 39.7 & 0.8 \\
\hline WW Aur & 1.88 & 6.603 & 3.903 & 11.2 & 0.5 & 11.9 & 1.1 \\
\hline RR Lyn A & 2.50 & 5.928 & 3.883 & 12.6 & 1.0 & 10.0 & 1.0 \\
\hline RR Lyn B & 1.93 & 6.878 & 3.860 & 11.7 & 1.1 & 10.0 & 1.0 \\
\hline ZZ Boo & 2.22 & 7.543 & 3.835 & 8.4 & 0.4 & 8.9 & 0.8 \\
\hline HS Hya A & 1.28 & 8.791 & 3.816 & 9.1 & 0.3 & 11.0 & 0.9 \\
\hline HS Hya B & 1.22 & 8.961 & 3.806 & 9.2 & 0.3 & 11.0 & 0.9 \\
\hline SZ Psc A & 1.40 & 8.746 & 3.809 & 8.6 & 1.5 & 11.3 & 0.9 \\
\hline SZ Psc B & 4.95 & 7.566 & 3.624 & 9.9 & 1.0 & 11.3 & 0.9 \\
\hline Z Her A & 1.85 & 7.664 & 3.809 & 10.7 & 0.5 & 10.2 & 0.8 \\
\hline Z Her B & 2.75 & 8.664 & 3.643 & 9.8 & 0.5 & 10.2 & 0.8 \\
\hline V1143 Cyg & 1.34 & 6.613 & 3.806 & 24.4 & 0.5 & 25.1 & 0.6 \\
\hline LX Per A & 1.64 & 8.913 & 3.787 & 7.5 & 0.4 & 10.0 & 1.0 \\
\hline LX Per B & 3.05 & 8.833 & 3.661 & 7.5 & 0.4 & 10.0 & 1.0 \\
\hline UX Men & 1.31 & 8.973 & 3.781 & 9.5 & 0.3 & 9.9 & 0.6 \\
\hline RT And A & 1.24 & 9.045 & 3.778 & 9.8 & 0.4 & 13.3 & 1.1 \\
\hline RT And B & 0.87 & 11.325 & 3.605 & 10.9 & 0.5 & 13.3 & 1.1 \\
\hline UV Psc A & 1.11 & 9.291 & 3.743 & 11.5 & 0.5 & 15.9 & 1.3 \\
\hline UV Psc B & 0.83 & 10.5 & 3.608 & 15.8 & 1.1 & 15.9 & 1.3 \\
\hline AR Lac B & 1.52 & 7.121 & 3.730 & 24.2 & 1.0 & 23.8 & 0.6 \\
\hline AR Lac B & 2.81 & 6.621 & 3.654 & 23.4 & 1.3 & 23.8 & 0.6 \\
\hline AR A & 1.78 & 6.8 & 3.973 & 7.7 & 0.4 & 8.2 & 0.8 \\
\hline AR Aur B & 1.82 & 6.941 & 3.972 & 7.2 & 0.4 & 8.2 & 0.8 \\
\hline $\mathrm{EI} \mathrm{Ce}$ & 2.90 & 8.1 & 3.851 & 4.6 & 0.3 & 5.0 & 0.6 \\
\hline EI Cep B & 2.33 & 8.686 & 3.841 & 4.6 & 0.3 & 5.0 & 0.6 \\
\hline V624 Her A & 3.03 & 6.629 & 3.893 & 7.2 & 0.4 & 6.9 & 0.7 \\
\hline V624 Her B & 3.23 & 7.429 & 3.816 & 6.7 & 0.4 & 6.9 & 0.7 \\
\hline UV Leo & 1.10 & 9.733 & 3.752 & 8.8 & 0.6 & 10.9 & 1.2 \\
\hline BH Vir A & 1.12 & 9.868 & 3.772 & 7.0 & 0.7 & 7.9 & 1.5 \\
\hline BH Vir B & 1.06 & 11.678 & 3.686 & 4.0 & 0.7 & 7.9 & 1.5 \\
\hline YZ Cas A & 2.53 & 5.747 & 3.944 & 11.0 & 0.6 & 11.2 & 0.6 \\
\hline YZ Cas B & 1.3 & 8.357 & 3.835 & 10.5 & 0.6 & 11.2 & 0.6 \\
\hline KW Hya A & 2.13 & 6.3 & 3.906 & 11.0 & 0.6 & 12.1 & 0.9 \\
\hline KW Hya B & 1.48 & 7.882 & 3.841 & 10.5 & 0.6 & 12.1 & 0.9 \\
\hline HD 209458 A & 1.15 & 7.653 & 3.764 & 21.4 & 0.6 & 21.2 & 0.9 \\
\hline$\beta$ Per A & 3.09 & 2.206 & 3.979 & 36.4 & 1.7 & 35.1 & 0.9 \\
\hline$\delta$ Lib A & 3.28 & 5.070 & 3.957 & 10.1 & 0.4 & 10.7 & 0.9 \\
\hline Sun $(25 p c)$ & 1.000 & 6.820 & 3.746 & 39.3 & 0.8 & $(40.0)$ & \\
\hline
\end{tabular}

measurements. Worek had a relatively short period for the third orbit at $2.762 \mathrm{yr}$, which can be checked from the Hipparcos observations that covered a $\sim 4$-yr time interval. Trials of this orbit have failed to produce an improvement in Hipparcos residuals, however, while there are other reasons to prefer a different one, for example, the new photometric solution indicates a significantly more massive and brighter companion star than Worek's. If we accept Worek's $\gamma$-velocities as of the right order, there would have to be an order of magnitude increase in the period of the third orbit to increase the mass-function to an appropriate value. A longer period, while able to reconcile the mass with the scale of radial velocity changes, would allow Hipparcos to fail to detect significant changes of 


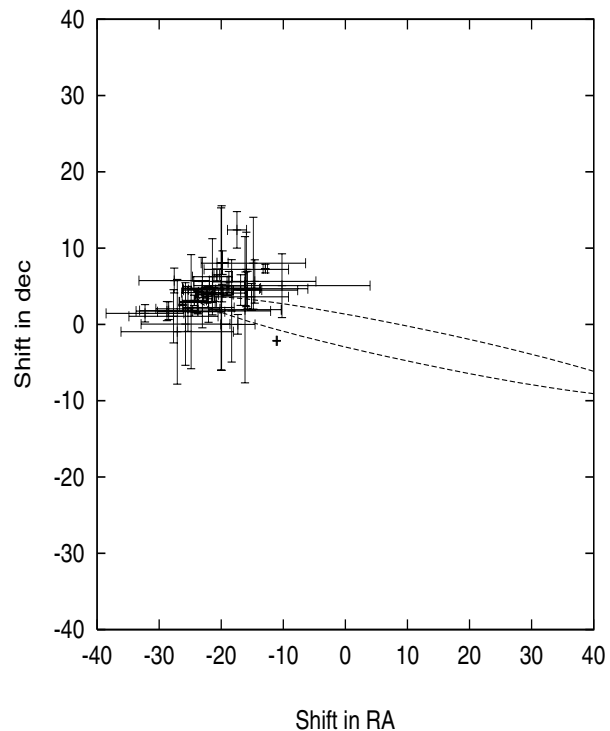

Figure 3. Plot of Hipparcos data trial fit to an orbit of $\delta$ Lib about a barycentre with a third body, corresponding to parameters given in Table 2 .

Table 2. Tentative parameters of the wide orbit of $\delta$ Lib corresponding to the fitting of $\mathrm{O}-\mathrm{C}$ residuals together with those of the Hipparcos abscissal measures.

\begin{tabular}{lccr}
\hline Parameter & Value & Parameter & \multicolumn{1}{c}{ Value } \\
\hline$P$ & $23.5 \mathrm{y}$ & Ep. & 2448347.75 \\
$a$ & $39.3 \mathrm{mas}$ & $e$ & 0.49 \\
$i$ & $80^{\circ} 3$ & $\omega$ & $317^{\circ}$ \\
$\Omega$ & $265^{\circ}$ & $\Delta \alpha$ & $17.1 \mathrm{mas}$ \\
$m_{c}$ & 0.48 & $\Delta \delta$ & $8.8 \mathrm{mas}$ \\
\hline
\end{tabular}

position, particularly if the third star was close to its apse at the time of observation. A longer period $(\sim 23.5 \mathrm{yr})$ is in keeping with the $\mathrm{O}-\mathrm{C}$ oscillation mentioned above.

In Fig. 3, we show the result of a trial fitting in which the period, projected semimajor axis, eccentricity and longitude values were taken from the provisional $\mathrm{O}-\mathrm{C}$ analysis, and inclination and nodal angle values were optimized from the Hipparcos astrometry, also allowing for a mean shift in the centroid of the orbit from the position given for $\delta$ Lib in the Hipparcos catalogue. The corresponding parameters are listed in Table 2. This approach is essentially similar to that of Ribas et al. (2001) for R CMa. The s.d. error of an individual residual is 1.8 mas in this fitting, which is close to typical Hipparcos solutions for fixed stars, so that this result compares with the no-orbit solution of the Hipparcos catalogue. The third body mass that would go with the light-time effect mass function, however, would be only $0.48 \mathrm{M}_{\odot}$ with such a high inclination, and this is inconsistent with the light curve model. Other areas of uncertainty about this have been noted by Budding et al. (2004a). VLBI radio observations could help probe this subject through its high accuracy positional information, as with the parallel case of $\beta$ Per (Lestrade et al. 1999). 

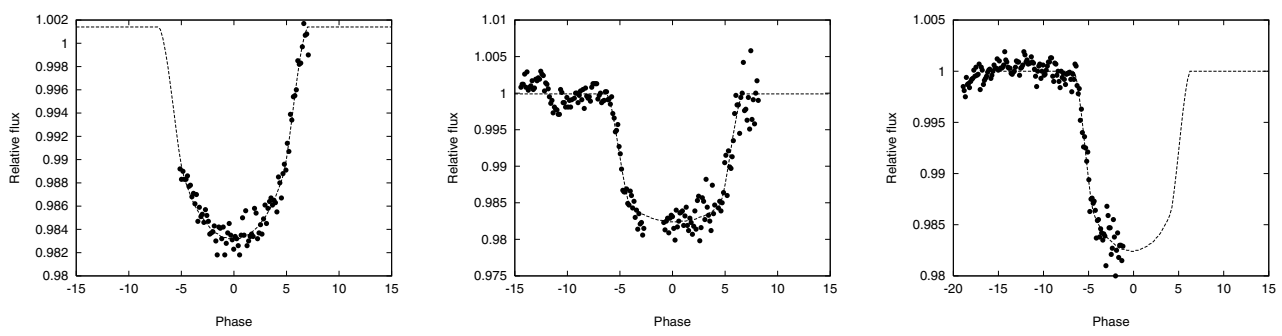

Figure 4. B light curves of three transits across the disk of HD 209458 by its planetary companion as observed by Sullivan \& Sullivan (2003). NB: the axes, scaled according to the data distribution of each set, are different for the three transits.

\section{Binaries containing very low mass components}

The circumstances of this meeting invite consideration of the cosmic frequency of planetary transits. The possibility that they may be more detectable than generally supposed was made by Demircan (private communication), noting the example of the Trojan asteroids in the solar system. The vanishing azimuthal gravity derivative in the restricted two-body problem at the Lagrangian equilateral points $\mathrm{L}_{4,5}$ allows matter accumulation, and, noticeably, this should be in the same plane as the two main bodies. This implies that sufficiently careful monitoring of classical eclipsing binaries around phases $\pm 60^{\circ}$ could reveal the existence of Trojan planets. A planet comparable to Venus would produce a light diminution of only $1 \mathrm{mmag}$. Current plans for satellite-based photometry, however, are specifying $10^{-4} \mathrm{mag}$ as a precision guideline (Luri et al. 2002; Giménez 2003). An interesting case of what a light curve of such an occurrence may resemble comes from the example of HD 209458. In Fig. 4, data of the 'planetary' transits in this system observed from Hawaii in 1999 and 2000 are presented.

Information on the circumstances of these observations was given by Jha et al. (2000). The general subject has been reviewed by Perryman (2000) and Black \& Stepinski (2003), while Sullivan \& Sullivan (2003) referred to http://www.obspm.fr/encycl/encycl.html as a useful website for up-to-date information. HD 209458 was one of a small number of candidate stars showing spectral evidence of low mass companions with reasonably short periods, some proportion of which would be likely to show eclipses. Slight light losses, interpreted as the transits of a planetary sized body, were first observed in September 1999 (Charbonneau et al. 2000) and these were confirmed from the Fairborn Observatory in November 1999 (Henry et al. 2000) a few days before our data was gathered at Mauna Kea.

The data shown in Fig. 4 were analysed using the CURVEFIT package, most recently reported on by Budding et al. (2004b). Speed of evaluation of an algebraic form of fitting function is an advantage when exploring a wide range of parameter space and evaluating the parameter error matrix. That this matrix corresponds, geometrically, to a closed ellipsoid is sufficient and necessary for formal determinacy of the underlying model. Examination of the error matrix is necessary in order to find out that the parametrization neither surpasses nor under-utilizes the information content of the data. Its evaluation is a key feature in this approach (cf. Banks \& Budding 1990). Each data set was fitted separately and the number of parameters to be optimized appropriately chosen, with initial guidelines coming from the papers of Henry et al. (2000) and Sullivan \& Sullivan (2003). We give the curve-fitting details in Table 3. Combining these relative parameters with the absolute data on the primary of HD 209458 given in Table 2 and using also the calibration equations (2.2) and (2.3) we derive the absolute parameters given in Table 4 . It may be noted that these values are within the error limits of those given by Brown 
Table 3. HD 209458 curve-fit details: optimal parameters and errors.

\begin{tabular}{crrrrrr}
\hline Parameter & \multicolumn{2}{c}{$15 / 11 / 99$} & \multicolumn{2}{c}{$12 / 11 / 00$} & \multicolumn{2}{c}{$19 / 11 / 00$} \\
\hline$U$ & 1.0012 & 0.0009 & 1.0002 & 0.0005 & 1.0003 & 0.0005 \\
$T_{0}$ & 1497.7993 & 0.001 & 1860.8471 & 0.0002 & 1867.8965 & \\
$r_{1}$ & 0.1165 & & 0.1159 & 0.007 & 0.1161 & \\
$r_{2}$ & 0.0140 & 0.0006 & 0.0149 & 0.0003 & 0.0147 & 0.0011 \\
$i$ & 86.9 & 0.4 & 86.1 & 0.7 & 86.1 & 0.9 \\
$u$ & 0.77 & 0.10 & 0.38 & 0.07 & 0.57 & 0.1 \\
$\Delta l^{\prime}$ & 0.001 & & 0.0018 & & 0.001 & \\
$\chi^{2} / \nu$ & 0.74 & & 0.97 & & 1.03 & \\
\hline
\end{tabular}

Table 4. Adopted Absolute Parameters for HD 209458.

\begin{tabular}{ccc}
\hline Parameter & Value & p.e. \\
\hline Period & $3.52474 \mathrm{~d}$ & $0.00001 \mathrm{~d}$ \\
Epoch (HJD) & 2451497.7993 & \\
$A$ & 9.837 & $0.04 \mathrm{R}_{\odot}$ \\
$R_{1}$ & 1.14 & $0.01 \mathrm{R}_{\odot}$ \\
$R_{2}$ & 1.40 & $0.03 \mathrm{R}_{\text {Jup }}$ \\
$i$ & 86.4 & $0.3^{\circ}$ \\
$u$ & 0.57 & 0.14 \\
$M_{1}$ & $1.03 \mathrm{M}_{\odot}$ & $*$ \\
$M_{2}$ & $0.62 \mathrm{M}_{\text {Jup }}$ & $*$ \\
$V_{\text {Abs }}$ & 4.31 & $*$ \\
$V$ & 7.653 & $*$ \\
$B-V$ & 0.594 & \\
$T$ & $5920 \mathrm{~K}$ & \\
Dist. & 46.7 & $0.8 \mathrm{pc}$ \\
\hline
\end{tabular}

* These values come from Henry et al. 2000

et al. (2001), while the mean limb-darkening coefficient $u$ is also within its error limit of modern theoretical estimates (e.g. 0.71 from van Hamme 1993).

\section{Concluding remarks}

Semeniuk (2000), following a Barnes-Evans flux calibration, pointed out that parallaxes obtained by the eclipse method could have an accuracy surpassing that of the Hipparcos programme if care was taken about the selection of suitable binaries. We have confirmed that the method can be extended, using the calibrations of Popper (1998), to similar MS-like stars, but making use of the $U B V$ system in which large numbers of objects have reliable measurement records. We propose also that the primaries of classical Algol systems can be included in the procedure, provided care is taken in establishing their radii and fluxes. Of course, only one star in $\sim 1000$ shows clear eclipses, but the fact that the effect can be discerned, in principle, out to as far as stars can be individually monitored gives the method a cosmic significance not shared by astrometric work of the present generation.

Eclipse data present other factors of relevant interest. For example, careful photometry over a wide spectral range, can allow detailed analysis to reveal the presence of companion objects to the eclipsing pair. This was strongly indicated in the case of $\delta$ Lib from $U B V J H K$ light curve fittings. The timing of eclipses can also indicate the presence of physical companions and even lead to estimates of a number of key parameters of the 
third orbit. In the case of nearby objects, Hipparcos observations can be reanalysed, using these parameters, to check on orbital solutions in order to find the remaining two elements not given by $\mathrm{O}-\mathrm{C}$ data. Since the absolute orbital scale can then be known, as well as its apparent size on the sky, the parallax directly follows.

Further checks of distances are possible when the stars happen to be radio sources, as was clearly shown by Lestrade et al. (1999) for Algol, and indirectly shown in the resolved map of the Algol source produced by Mutel et al. (1998). Slee et al. (1987) listed a few dozen stars that may fall into the category of having VLBI-determinable parallaxes.

Analysis of the B observations of HD 209458 was carried out to elicit parameter resolution for ground-based photometry, but of higher accuracy than typical. It is worth noting that submillimag accuracy is achievable even with a $0.6-\mathrm{m}$ telescope and $2 \mathrm{~min}$ integrations from a ground-based site, provided that site is suitably located: in this case at an altitude of $\sim 4000 \mathrm{~m}$. On this basis, hour-long integrations with a $>1-\mathrm{m}$ telescope from similar locations should allow $\mu$ mag accuracies to be approachable for brighter stars. HD 209458 turns out to be high up on the list of nearby eclipsing binaries. In the well-studied lists from Popper (1998) and Semeniuk (2000), it comes in fifth, after the very well-known stars $\beta$ Aur, $\beta$ Per, V1143 Cyg and AR Lac, and may be the nearest simple binary with complete eclipses. $\dagger$ This point alone seems a strong indication of the likely high relative frequency of such systems cosmically.

Taking these last two points together, a project to check for less than Jupiter-sized bodies accompanying the brightest stars seems feasible even with ground-based instruments. One possible initial set of targets could come from checking, with near $\mu$ mag accuracy, the $60^{\circ}$ phase regions of the nearest eclipsing binaries. From one point of view, it may be unfortunate that a relatively high proportion are those nearby sunlike binaries, such as UV Psc, RT And and BH Vir, whose light curves are known to be complicated by activity effects. On the other hand, this could simply give an extra reason motivating a prolonged photometric survey. But if complication-free binaries are to be preferred, after V1143 Cyg, whose eclipses are still too shallow to ensure a suitable degree of coplanarity, probably the best candidate would be the 'textbook' example YZ Cas. Otherwise, candidates should probably come from the more general arguments proposed by Henry et al. (2000) and others.

\section{Acknowledgements}

E.B. would like to acknowledge useful comments and support from Prof. O. Demircan and the Physics Dept., 18th March University of Canakkale, Turkey.

\section{References}

Banks, T. \& Budding, E. 1990 Ap\&SS, 167, 221.

Barnes, T.G. \& Evans, D.S. 1976, MNRAS, 174, 489.

Black, D.C., \& Stepinski, T.F. 2003, Proc. 8th IAU Asian-Pac. Reg. Meet., eds. S. Ikeuchi et al., Astron. Soc. Pacific, 77.

$\dagger$ We are grateful to J. Southworth for drawing attention to the case of $\alpha \mathrm{CrB}$. Tomkin \& Popper's (1986) solution, for the primary at least, yields a parallax $42.2 \pm 0.3$ mas, very close to that of $\beta$ Aur. Its Hipparcos parallax $43.7 \pm 0.8$ mas indeed surpasses the latter. The completely eclipsing model used by Tomkin \& Popper discarded a relatively large number of eclipse observations, however, and the resulting model for the (non-MS) secondary produces discordant results (e.g. a photometric parallax of $49 \pm 3$ mas). It thus seems safer to follow Tomkin \& Popper's suggestion of waiting for the difficult, but worthy, task of producing definitive light curves to be completed before including $\alpha \mathrm{CrB}$ among the reference stars. 
Brown, T.M., Charbonneau, D., Gilliland, R.L., Noyes, R.W. \& Burrows, A. 2001, ApJ, 552, 699.

Budding, E. 1993, Introduction to Astronomical Photometry, Cambridge Univ. Press, Cambridge

Budding, E. \& Zeilik, M. 1987, ApJ, 319, 827.

Budding, E. et al. 2004a, Ap\&SS, in press.

Budding, E., Rhodes, M., Priestley, J. \& Zeilik, M. 2004b, Astron. Nachr., 325, 433.

Charbonneau, D., Brown, T.M., Latham, D.W., \& Mayor, M. 2000, ApJ, 529, L45.

ESA 1997, Tycho Catalogue (ESA SP-1200), 542.

Giménez, A. 2003, Publ. Canakkale Onsekiz Mart Univ. (Astrophys.), 3, 19.

Henry, G.W., Marcy, G.W., Butler, R.P., \& Vogt, S.S. 2000, ApJ, 529, L41.

Jha, S., Charbonneau, D., Garnavich, P.M., Sullivan, D.J., Sullivan, T., Brown, T.M. \& Tonry, J.L. 2000, ApJ, 540, L45.

Kopal, Z. 1959, Close Binary Systems, Chapman \& Hall, London \& New York.

Lacy, C.H. 1978, Ph.D. Thesis, Univ. Texas, Austin.

Lacy, C.H. 1979, ApJ, 228, 817.

Lazaro, C., Arevalo, M.J. \& Claret, A. 2002, MNRAS, 334, 542.

Lestrade, J-F. et al. 1999, A\&A, 344, 1014.

Luri, X., Figueras, F. \& Torra, J. 2002, EAS Publ. Ser., eds. O. Bienaymé and C. Turon, EDP Sciences, 2, 171.

Malkov, O.Y. 1993, Bull. Inf. Centre Donn. Stellaires, 42, 27.

Mutel, R.L., Molnar, L.A., Waltman, E.B. \& Ghigo, F.D. 1998, ApJ, 507, 371.

Nordström, B. \& Johansen, K.T. 1994, A\&AA, 291, 777.

Perryman, M.A.C. 2000, Rep. Prog. Phys., 63, 1209.

Popper, D.M. 1998, PASP, 110, 919.

Ribas, I., Arenou, F, \& Guinan, E.F. 2002, AJ, 123, 2033.

Russell, H.N. 1948, Centennial Symposia ed. C. Payne-Gaposchkin, Harvard University Press, Cambridge, Mass.

Semeniuk, I. 2000, Acta. Astron., 50, 381.

Slee, O.B., Nelson, G.J., Stewart, R. T., Wright, A.E., Innis, J.L., Ryan, S.G., \& Vaughan, A.E. 1987, MNRAS, 229, 659 .

Sullivan, D.J. \& Sullivan, T. 2003, Baltic. Astron., 12, 145.

Tomkin, J. \& Popper, D.M. 1998, AJ, 91, 1428.

van Hamme, W. 1993, AJ, 106, 2096.

Wilson, R.E. and Devinney, E.J. 1971, ApJ, 166, 605.

Worek, T.F. 2001, PASP, 113, 964.

\section{Discussion}

Dimitri Pourbaix: In your data for the $\delta$ Librae system you have looked for a third body over a period of 23 years. Have you checked that the Hipparcos proper motion is discrepant with respect to the Tycho- 2 proper motion? Because if the period is indeed that long with respect to the length of the mission, then you should see a departure of the two proper motions. One based on just 3 years and one based on 100 years.

ED Budding: Yes, I'm aware of that technique. This approach has come from the study of Ribas et al. [2002, AJ, 123, 2033] on R CMa. The great emphasis in that paper is on the apparent shift in position. They had to sum the proper motions down to just 4 individual points in order to get error bars that gave a significant effect. Given that you have only got at most a 4-year time interval in a 23-year orbit, the change of the speed on the sky is going to be a bit small. You can certainly get the mean value from a century of observation. The very long-term observations - going back a 100, 150 years - have appreciable errors on the milli-arcsecond scale. 
Dimitri Pourbaix: During the 3 years you will see essentially the orbiter motion, so what is quoted as the proper motion in the Hipparcos catalogue is likely to be confused with the orbiter motion. Whereas in the 100 year period of the proper-motion-based Washington catalogue, you would get just a proper motion, so you should see a departure of the Hipparcos proper motion with respect to that proper motion.

ED BudDing: I'm not sure how much that departure differs within the scale of the error bars involved. I have not checked this in detail, as we wanted to concentrate first on the possible positional orbit. We've found one, but I think there could be more. This isn't necessarily a unique solution for this star.

Dimitri Pourbaix: No, but if you take the Tycho-2 proper motion, that would give you two parameters, thus giving more chance to detect your actual orbiter motion. So you would reduce the number of consistent orbiter solutions.

John SouthworTh: About HD 209458 being near the star with complete eclipses: $\alpha$ CrB shows complete eclipses - about 0.05 mag deep. It might be closer, but it is too bright for modern telescopes, so good luck observing it.

ED BudDInG: It wasn't on my list. The four which I found that were nearer were $\beta$ Aur, Algol, AR Lac and one other, V(some-big-number) Cyg. Those came from Popper's list and Semeniuk's list.

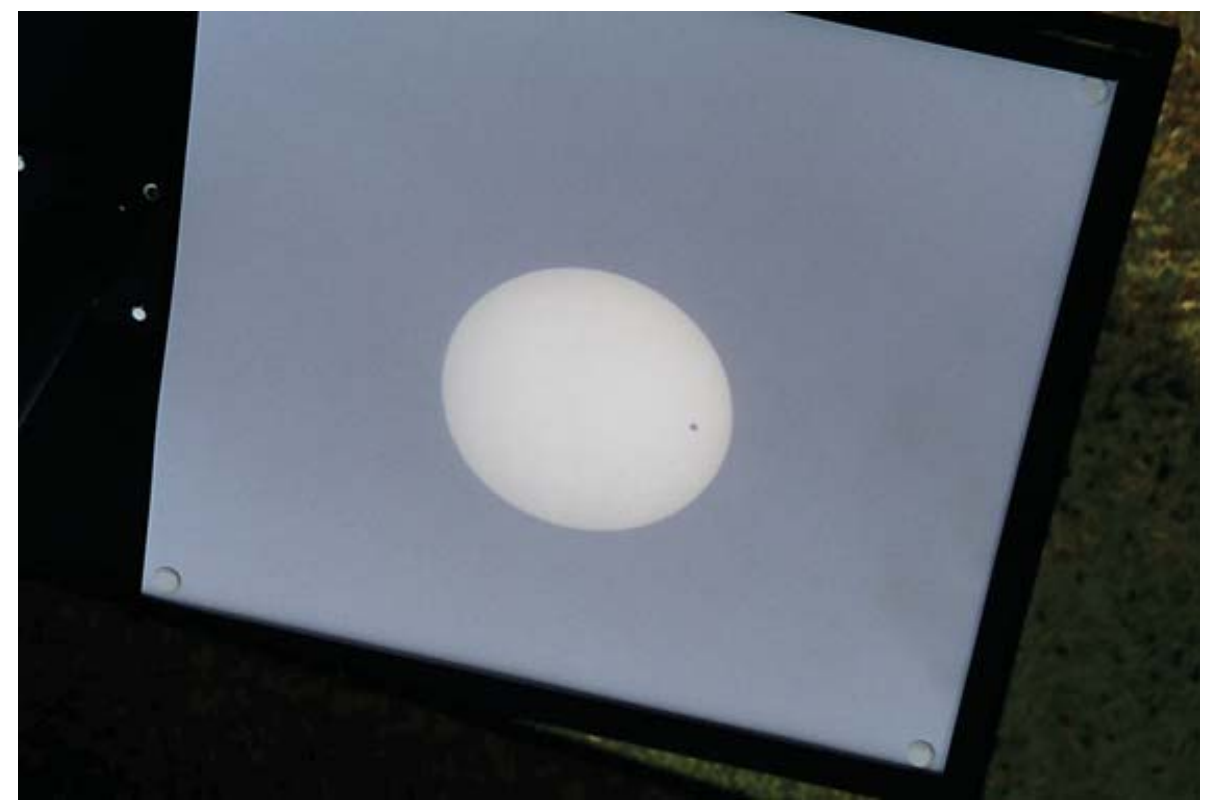

